\title{
Aplicación de biofertilizantes foliares en el cultivar Huaycha (Solanum tuberosum subsp. andigena) en los valles interandinos de Bolivia
}

\author{
E. Mamani ${ }^{1}$, V. Morales ${ }^{2}$, N. Ortuño ${ }^{1 / *}$
}

Recibido: $23 / 06 / 2016$

Aceptado: 13/11/2016

Accessible en linea: Diciembre 2016

\section{Resumen}

Por la necesidad de disponer tecnología alternativa e inducir a una producción saludable a los pequeños productores, se evaluaron diferentes tipos de biofertilizantes foliares, conocidos como bioles. Se estableció el ensayo en Pocona-Cochabamba (2400 a 3800 m.s.n.m.), utilizando tres biofertilizantes Bioagroec simple, Bioagroec compuesto, Fertisol y un testigo como maneja el agricultor, cada uno combinados con tres aplicaciones . Bajo las condiciones de estudio la altura de la planta de papa se incrementó en un $10 \%$ con el Fertisol, el número de tubérculos se incrementó en $42 \%$ con Bioagroec simple. Asimismo, en el número de tubérculos para la categoría chilimurmu (última categoría en tamaño) fue $50 \%$ más con Bioagroec simple, todos comparados respecto al testigo (sin aplicación). En la frecuencia de aplicación solo se detectó diferencias con la aplicación del Fertisol con tres aplicaciones, el que mejoró el rendimiento en número de tubérculos en la categoría chapara (categoría mayor en tamaño) en $33 \%$ más. El rendimiento en peso se incrementó $45 \%$ al aplicar Bioagroec simple (B2), respecto al testigo (sin aplicación). El rendimiento en peso de tubérculos de tamaño cuarta (penúltima en tamaño) se incrementó $86 \%$ con Bioagroec simple, respecto al testigo (sin aplicación). En el análisis económico, el biofertilizante (B2: con dos aplicaciones), reportó los mayores ingresos netos (US\$ $1553.638 \mathrm{ha}^{-1}$ ) y el menor costo variable (US\$22.65ha $\mathrm{h}^{-1}$ ). Mostrando una tasa de retorno marginal de $41.81 \%$.

Palabras claves adicionales: Biofertilizantes, producción orgánica, rendimiento, fertisol.

\section{Application of foliar bio-fertilizers in the Huaycha cultivar (Solanum tuberosum subsp. andigena) in the inter-Andean valleys of Bolivia}

\section{Summary}

Due to the need to provide alternative technology and to induce a healthy production to the small producers, different types of foliar bio-fertilizers, known as bioles, were evaluated. The trial was established in Pocona-Cochabamba (2400-3800 m.a.s.1.), by using three biofertilizers: simple Bioagroec, compound Bioagroec, Fertisol and a witness to check how the farmer handles them, each one combined with three applications. Under the study conditions, the height of the potato plant increased by $10 \%$ with Fertisol, the number of tubers increased by $42 \%$ with simple Bioagroec. Also, the number of tubers for the chilimurmu category (last category in size) was 50\% more with simple Bioagroec, all

\footnotetext{
* Autor para correspondencia: noelortunocastro@ gmail.com

1 Universidad Mayor de San Simón. Facultad de Ciencias Agrícolas y Pecuarias "Martín Cárdenas", Cochabamba, Bolivia.

2 AGROECO, Cochabamba, Bolivia.
} 
compared according to the witness (without application). In the frequency of application, only differences were detected with the application of Fertisol with three applications, which improved the yield in number of tubers in the category Chapara (category major in size) by $33 \%$ more. The yield by weight was increased by $45 \%$ when applying Bioagroec simple (B2), with respect to the witness (without application). The weight yield of tubers of fourth size (penultimate in size) was increased by $86 \%$ with Bioagroec simple, with respect to the witness (without application). In the economic analysis, the biofertilizer (B2: with two applications) reported the highest net income (US\$1553.638 ha ${ }^{-1}$ ) and the lowest variable cost (US\$22.65 $\mathrm{ha}^{-1}$ ), showing a marginal rate of return of $41.81 \%$.

Additional keywords: Bio-fertilizers, organic production, yield, fertisol.

\section{Introducción}

En la actualidad, en Bolivia y en el mundo se buscan nuevas alternativas para un manejo sostenible y racional en la producción agrícola, frente al uso excesivo de fertilizantes plaguicidas y la necesidad de incrementar los rendimientos y recursos económicos de los agricultores. El uso inadecuado de fertilizantes químicos ha deteriorado el ambiente y el suelo; además, que representan altos costos para los productores siendo necesario incursionar en el uso de técnicas y conocimientos que permitan reducir los costos de producción (Adekiya y Agbede, 2009).

Los biofertilizantes o biopreparados se originan a partir de la fermentación y descomposición aeróbica y termofilica de materiales orgánicos, tales como estiércoles de animales, plantas verdes, frutos, etc. (Blanco, 2003 y Aguilar 2005) $\mathrm{y}$ por medio de microorganismos se descomponen y después de un tiempo pueden ser aplicados directamente sobre los cultivos vía foliar o sobre los suelos durante el riego. Se puede fabricar una gran variedad de biofertilizantes que van desde el más sencillo hasta el mas complejo enriquecido con algunos minerales, cenizas y harinas complementarias (Las ventajas y los resultados más comunes que se logran con los biofertilizantes en los cultivos (Ramirez, 2002) son:
- Aumento de la resistencia contra el ataque de insectos y enfermedades.

- El aumento en la precocidad en todas las etapas del desarrollo vegetal de los cultivos (incremento del número de cosechas por año).

- La longevidad de los cultivos perennes es mayor y al ser tratados con los biofertilizantes se recuperan más rápidamente del stress post- cosecha y pastoreo.

- El aumento en la cantidad, la uniformidad, tamaño y la calidad nutricional; el aroma y el sabor de lo que se cosecha y se conserva por un periodo más prolongado, principalmente frutas y hortalizas.

El Biol o biofertilizante foliar es considerado como fito-estimulante, al ser aplicado al suelo o al follaje, permite aumentar el volumen de raíces y hojas, mejorando la capacidad fotosintética de las plantas, mejorándose así la producción y la calidad de las cosechas (Pomacosi, 1994). Los abonos foliares tienen la particularidad de incorporar nutrientes de fácil asimilación a través del follaje introduciéndose macro y micro nutrientes (Villarroel, 1999). Los efectos positivos logrados al aplicar los biofertilizantes, en los diferentes cultivos, inciden en el rendimiento.

La composición química del biofertilizante o biol, deriva básicamente 
de la fermentación anaeróbica del estiércol del ganado lechero, donde intevienes microorganismos naturalmente habitan el estiércol, el resultado de este proceso es considerado una fuente de fitorreguladores que promueven el crecimiento de los vegetales (Capuli et al., 2007).

Se denomina fitorreguladores a los compuestos orgánicos que en pequeñas cantidades inhiben, promueven, modifican algún proceso fisiológico. Estas son sustancias elaboradas en base a hormonas vegetales naturales $\mathrm{o}$ de bioactivos sintéticos que al ser aplicados en pequeñas dosis, regulan, estimulan o detienen el crecimiento de las plantas. La fitorregulación es hacer que la planta se desarrolle en forma correcta y normal, aunque las condiciones ambientales sean adversas y para lograr este fin, debe operar con moléculas iguales o muy similares a las que se encuentran en la planta de modo natural (Medina, 1992).

Dentro de éste marco se propone el uso de abonos orgánicos concretamente la elaboración y aplicación de biofertilizantes, son abonos líquidos fermentados a partir del estiércol de aplicación foliar, rico en macro y micro elementos restituye la armonía mineral en el suelo mejorando sustancialmente su fertilidad por tanto puede constituirse en una alternativa de fertilización complementaría de cultivos reduciendo por tanto los costos de producción e incrementando beneficios a favor de los agricultores.

El objetivo de este trabajo es evaluar el efecto de "Biofertilizantes foliares" o bioles en el desarrollo y rendimiento del cultivo de la papa, variedad Waych'a en temporada de siembra "mishka" (siembra de invierno), con el fin de disminuir costos de producción y aumentar las ganancias de los agricultores. Se determinó el efecto y frecuencia óptima en la aplicación de tres tipos de Biofertilizantes o bioles (Bio Agroec
Compuesto, Bio Agroec Simple y Fertisol), comparando tres niveles (dos, tres y cuatro aplicaciones) en dosis del 5 $\%$ (1 1 . / 20 lt. de agua) en el desarrollo y rendimiento del cultivo de la papa Waych'́a en la comunidad de Pocona Departamento de Cohabamba. Complementariamente se hizo una evaluación económica en relación al rendimiento y calidad de tubérculo,

\section{Materiales y métodos}

El trabajo se realizó en la comunidad de Pocona en el Centro de formación y capacitación en agroecología AGROECO, en el municipio de Pocona, Tercera Sección de la provincia Carrasco, en el departamento de Cochabamba. La investigación se realizó durante el periodo que corresponde a la siembra "mishka" siembra de invierno, en los los meses de agosto a diciembre. Geográficamente se ubica en la región tropical entre los $17^{\circ} 29^{\prime}-17^{\circ} 47^{\prime}$ de latitud sur y $65^{\circ} 15^{\prime}-65^{\circ} 34^{\prime}$ de longitud oeste. El territorio del municipio comprende una altitud entre los 2400 y $3800 \mathrm{msnm}$ (Morales, 2005). Su paisaje predominantemente montañoso presenta una topografía ondulada de pendientes moderadas alternadas por planicies aluviales (Morales, 2005).donde la precipitación promedio es de $612.55 \mathrm{~mm}$. /año, La temperatura media anual es de $17.45^{\circ} \mathrm{C}$, presentándose extremos de $-5^{\circ} \mathrm{C}$ en junio y julio y máximos extremos de $281^{\circ} \mathrm{C}$, entré los meses de octubre y noviembre. La humedad relativa del ambiente fluctúa entre $39 \%$ y $66 \%$. La evapotranspiración potencial está estimada en 1265 mm/año.

Según el análisis del suelo, el nitrógeno y el potasio son deficientes Villarroel (1988), para el cultivo de papa, para el cual se recomienda de 0.20 de $\mathrm{N} \%$ y 0.51 (me/100 g) de potasio, sin embargo el contenido de fósforo se la considera alto. En relación al contenido de materia 
orgánica lo óptimo se considera un nivel de $3 \%$, el contenido de estos suelos es de $1.98 \%$ e indica un suelo bajo en contenido de materia orgánica. Por esta razón se enviaron muestras al azar del terreno donde se realizó la investigación al laboratorio de suelos de Facultad de Ciencias Agrícolas de la Universidad Mayor de Simón.

En la investigación se utilizó semilla de papa variedad Huaycha, Categoría Registrada y los siguientes tipos de bioles:

- Bioagroec compuesto ( elaborado por AGROECO)

- Bioagroec simple (elaborado por AGROECO)

- Fertisol (elaborado por la Fundación PROINPA)

\section{Preparación de biofertilizantes y su evaluación}

Los biofertilizantes que se aplicaron se elaboraron según los métodos utilizados por Restrepo (2002), y Agrecol Andes, enriquecido con sales minerales y leguminosas. El Fertisol preparado según Ortuño et al. (2011), tal como se indica en la Tabla 1.

Para el manejo de los datos se consideraron dos factores:

1- Biofertilizantes (Bioagroec compuesto $=\mathrm{B} 1), \quad($ Bioagroec simple $=$ B2), (Fertisol= B3).

2- Frecuencias de aplicación (F2= 2 aplicaciones 12 y 24 días después del primer aporque (DPA)), $\quad(\mathrm{F} 3=3$ aplicaciones 12, 24, y 36 días DPA), ( $\mathrm{F} 4=4$ aplicaciones cada 12, 24, 36 y 48 días DPA). Junto a un testigo (B0F0 $=$ sin aplicación), la dosis de aplicación equivale a 1 lt./mochila de 20 de litros de agua $(5 \%)$. El primer aporque correspondió a los 54 días.
Con los siguientes tratamientos: $\mathrm{T} 1=\mathrm{B} 0 \mathrm{~F} 0, \quad \mathrm{~T} 2=\mathrm{B} 1 \mathrm{~F} 2, \quad \mathrm{~T} 3=\mathrm{B} 1 \mathrm{~F} 3$, $\mathrm{T} 4=\mathrm{B} 1 \mathrm{~F} 4 ; \quad \mathrm{T} 5=\mathrm{B} 2 \mathrm{~F} 2, \quad \mathrm{~T} 6=\mathrm{B} 2 \mathrm{~F} 3$, $\mathrm{T} 7=\mathrm{B} 2 \mathrm{~F} 4, \quad \mathrm{~T} 8=\mathrm{B} 3 \mathrm{~F} 2, \quad \mathrm{~T} 9=\mathrm{B} 3 \mathrm{~F} 3 \quad \mathrm{y}$ $\mathrm{T} 10=\mathrm{B} 3 \mathrm{~F} 4$.

El diseño experimental que se utilizó fue un diseño de bloques completos al azar con factorial incompletó, con diez tratamientos (efectos simples e interacció biofertilizantes y frecuencia de aplicación) con tres repeticiones. La unidad experimental fue de $3 \mathrm{~m}$. de ancho x $3 \mathrm{~m}$. largo, y la unidad de muestreo fue de $2.4 \mathrm{~m} 2$. la superficie total del ensayo fue de $270 \mathrm{~m} 2$. Para el análisis estadístico de las variables de respuesta, se realizó un ajuste por medio de un modelo lineal (REPEATED) que nos permitió analizar la relación que existe con otros tratamientos.

Las variables evaluadas para alcanzar los objetivos planteados son: Porcentaje de emergencia, altura planta, número de tallos, número de tubérculos y rendimiento en peso.

Se complementó el trabajo con el análisis económico de los rendimientos, y se efectuó con el método de presupuestos parciales (Blajos y Quiroga, 1996).

\section{Resultados y discusión}

Los tratamientos recibieron condiciones iguales desde la preparación de los suelos, labores culturales (aporque, riego y control de plagas y enfermedades).

\section{Análisis químico de los biofertilizantes}

En general el análisis químico del laboratorio indicó que el $\mathrm{pH}$ varió de 4.7 a 4.2, valores que se consideran ácidos y que pueden ser la causa en el bloqueo de asimilación de nutrientes (Villarroel 1986). Respecto a los macroelementos y cationes intercambiables estuvieron dentro el requerimiento del cultivo, debido a que no se observaron síntomas 
de deficiencias o fitotoxicidad en el cultivo de papa, en las condiciones de estudio

\section{Desarrollo del cultivo}

Para determinar el efecto de los biofertilizantes durante el desarrollo en el cultivo de la papa, se evaluaron las siguientes variables:

\section{Porcentaje de Emergencia}

En el carácter porcentaje de emergencia no se identificaron efectos significativos (p>0.05), para los dos factores, Biofertilizantes (B0, B1, B2 y B3) y frecuencias (0, 2, 3 y 4), de aplicaciones, es decir el efecto de los biofertilizantes es el mismo en el porcentaje de emergencia.

\section{Número de tallos por planta}

En el número de tallos no se observaron diferencias significativas ( $p>0.05)$, entre los biofertilizantes (B0, B1, B2 y B3) ni en las frecuencias $(2,3$, y 4$)$ de aplicaciones. Es decir los efectos fueron similares para todos los tratamientos en esta variable.

\section{Altura planta}

La altura planta fueron analizados los datos de dos maneras, primero a los 60 dñias después de la siembra (Figura 1) y durante el crecimiento de toda la planta (Figura 2).

Se estimaron diferencias significativas entre biofertilizantes $(\mathrm{p}=0.0219)$, sobre la altura de la planta. Dada la situación se procedió a realizar comparación de medias de los biofertilizantes (B1, B2 y B3), respecto al testigo (sin aplicación). En el factor frecuencias no se estimaron diferencias ( $p>0.05)$.

Comparativamente las medias de altura planta (Figura 1), de los biofertilizantes frente al testigo, indica que el B3 muestra diferencias significativas $(\mathrm{P}=0.03880)$, de 72.18 a $79.7 \mathrm{~cm}$. Sin embargo el B1 y el B2 frente al testigo reportaron medias de 76.05 y $72.15 \mathrm{~cm}$. respectivamente en altura por planta, estadísticamente no apreciables respectó al testigo ( $>>0.05)$, esto muestra que el Fertisol favorece en el crecimiento foliar del cultivo de la papa en un $10 \%$ más respectó al testigo (sin aplicación).

El crecimiento de la planta a través del tiempo se muestra en la figura 2 , donde se aprecian los efectos producidos por los biofertilizantes durante el crecimiento en altura planta respecto al testigo, cuando se aplicaron 2, 3 y 4 veces en el transcurso del ciclo vegetativo del cultivo de papa.

La figura 2, muestra la altura de planta través del tiempo en el cultivo de papa, para eso se ajustaron los datos a una curva polinomial de segundo grado. Ahí se observan los efectos de los Biofertilizantes (B1 y B3), donde se van diferenciando, siendo mayor en la cuarta y quinta lectura, donde el B3 alcanzó mayor altura respecto a los tratamientos B1, B2 y testigo, habiéndose detectado diferencias estadísticas $(\mathrm{p}=0.03880)$.

Sin embargo, el incremento en altura planta del tratamiento B3 no se refleja en el rendimiento de tubérculos, siendo que el B2 reportó mayor peso y número en el rendimiento de tubérculos, pero no fueron diferencias significativas en altura planta.

Por los datos observados, es importante la frecuencia de aplicación de biofertilizantes $\mathrm{o}$ bioles, porque se obtienen mejores resultados hasta con tres aplicaciones, en sus diferentes preparados, en el desarrollo de las plantas, lo cual coincide con estudios realizados por Medranda Vera et al. (2016) y Céspedes (2016), en cultivos de pimiento y de pasto Nacella sp., respectivamente, obtuvieron mayores alturas y mas vigor en las plantas cuando aplicaron de dos a tres veces bioles de fermentación anaeróbica. 


\section{Cosecha}

En la cosecha se evaluó las variables rendimiento en número y peso que inciden en forma directa en los beneficios netos que se obtuvo a partir del uso de estos biofertilizantes.

\section{Rendimiento en número de tubérculos}

El rendimiento en número de tubérculos mostró diferencias solo para el factor biofertilizantes $(p=0.0062)$, y no así en las frecuencias de aplicación. Comparativamente las medias de rendimiento en número de tubérculos (Figura 3) indican que el tratamiento B2 muestró diferencias significativas ( $\mathrm{p}=0.0456)$, frente al testigo, con una media de 27 versus 19 número de tubérculos/ m2, es decir una diferencia de $42 \%$ más.

\section{Rendimiento en número de tubérculos por categoría}

En el carácter número de tubérculos por categorías, se estima diferencias $(\mathrm{p}=0.0316)$ para el factor biofertilizantes (B0, B1, B2 y B3), únicamente en la categoría chilimurmu, respecto a las otras categorías no se aprecio diferencias ( $p>0.05)$. En el factor de frecuencias (2, 3 y 4), de aplicaciones de los biofertilizantes se detectaron diferencias $(\mathrm{p}=0.0456)$, únicamente para la categoría chapara (tubérculos grandes).

Dada la situación se procedió a realizar comparación de medias de los biofertilizantes (B1, B2 y B3), respecto al testigo (B0). En las frecuencias de aplicaciones, se realiza un análisis individual para determinar cual de ellos tiene efecto.

\section{Frecuencias de aplicaciones en la categoría chapara (tamaño mayor)}

En el análisis individual por número de tubérculo, se detectaron diferencias significativas $(p=0.0078)$, únicamente en las frecuencias de aplicación del tratamiento $\mathrm{B} 3$ con $(2, \quad 3 \quad$ y 4 aplicaciones), donde las aplicaciones tienen efecto de tendencia cuadrática, éste incrementa fue de 1.6 a $5.1 \mathrm{n}^{\circ}$ de tub/m2, cuando se aplicó 2 y 3 veces el producto Bioagroec. Pero cuando se aplicó cuatro veces el B3 (Fertisol), se observó que el rendimiento baja $3.4 \mathrm{n}^{\mathrm{o}}$ de tub/ $\mathrm{m} 2$, para esta categoría.

\section{Chilimurmu (tubérculos más pequeños)}

Comparativamente las medias mostraron al tratamiento B2 (Bioagroec) con diferencias significativas $(\mathrm{p}=0.0204)$, frente al testigo, con un rendimiento de 8.7 versus 5.1 en $\mathrm{n}^{\mathrm{o}}$ de tubérculos $/ \mathrm{m} 2$, respectivamente. Es decir que el tratamiento B2 favorece a la producción más de los tubérculos mas pequeños en $50 \%$, respecto al testigo.

\section{Rendimiento Total en Peso}

Comparativamente las medias de rendimiento total en peso (Figura 4), indican que el tratamiento B2 muestra diferencias estadística $(\mathrm{p}=0.001)$, con un promedio de 16.4 a 11.09 t/ha, respectó al testigo, es decir que el Bioagro reportó 45 $\%$ más de rendimiento total respectó al testigo, bajo las condiciones de estudio.

\section{Rendimiento total en peso por categoría}

En el rendimiento total en peso por categorías se estimó diferencias $(\mathrm{p}=0.001)$, únicamente para el factor biofertilizante en la categoría murmu, en las otras categorías no se apreciarondiferencias estadísticas $(\mathrm{p}>0.05)$. Luego se realizaron comparaciones de medias de los tres biofertilizantes (B1, B2 y B3), respectó al testigo (B0), en la categoría murmu.

\section{Categoría Murmu}

En la comparación de medias se estima diferencias $(\mathrm{p}=0.0116)$, para el B2 únicamente con $5.4 \mathrm{t} / \mathrm{ha}$, frente al testigo 2.9 t/ha. Es decir con la aplicación del 
Bioagro, se detectó $86 \%$ más en la producción de la categoría murmu, respectó al testigo.

\section{Relación entre el rendimiento total en peso, emergencia, número de tallos, altura planta y número de tubérculos}

En el análisis de correlación realizado se estimo una relación positiva y altamente significativa $(\mathrm{p}<0.01)$, entre la variable rendimiento total en peso, número de tubérculos, y altura planta, lo que significa que la variable de rendimiento total en peso es dependiente del número de tubérculos y altura planta. Es decir a mayor número de tubérculos y altura de planta el rendimiento en general será mayor.

En el cultivo de papa, al aplicar al follaje los bioles es evidente un mayor desarrollo y mayor rendimiento de tubérculos grande o mayor número de tubérculos pequeños, como lo indican Garnica (1996) y Nina (2004) y eso puede variar con los insumos que se preparan (IICA, 2004 y Aguilar, 2005), como son el Fertisol o el Bioagrec simple y compuesto. También Macías et al., (2012) encontraron mayores rendimiento en el cultivo de pimiento cuando aplicaron biol al follaje.

\section{Análisis económico}

En la Tabla 2, se presentan los costos de producción y costos variables que intervienen en la producción del cultivo de la papa, los beneficios netos y la tasa de retorno marginal, respectivamente. Donde se muestra que los mayores ingresos netos por encima de $1000 \$$ por ha. se obtuvieron con el uso del Bioagroec simple (B2) y Fertisol (B3), con frecuencias de (dos, tres y cuatro) aplicaciones, respectó al testigo (sin aplicación).

En el análisis de dominancia T1 (B0=testigo) y $\mathrm{T} 2 \quad(\mathrm{~B} 2$, con 2 aplicaciones), son los tratamientos no dominados indicando ser los de menor costo variable y mayor beneficio neto, presentando una tasa de retorno marginal de $4181 \%$. De acuerdo al método propuesto por Blajos y Quiroga (1996), indican que por cada $22.65 \$$ invertidos en la producción de cultivo de papa, se recupera $41.81 \$$ adicionales. En base a éstos resultados se recomienda la aplicación del B2 con una frecuencia de dos aplicaciones cada 12 días después del primer aporque en el cultivo de la papa, siendo la única que presenta utilidades altas.

Bajo las condiciones de estudio en el cultivo de papa en el municipio de Pocona se constató, que la altura planta se incrementó en un $10 \%$ más cuándo se aplicó el Fertisol (B3), respectó al testigo ( $\sin$ aplicación), en el número de tubérculos se incrementó en un $42 \%$ más cuándo se aplicó el Bioagroec simple (B2), respectó al testigo (sin aplicación). Asimismo, en el número de tubérculos para la categoría chilimurmu éste se incrementa en un $50 \%$ más cuándo se aplicó el Bioagroec simple (B2), respectó al testigo (sin aplicación).

\section{Conclusiones}

En las frecuencias de aplicación de los biofertilizantes se estimaron diferencias con la aplicación del Fertisol (B3), con tres (F3), veces para el rendimiento en número de tubérculos en la categoría chapara (tubérculos grandes), en un $33 \%$ más respectó a las frecuencia cuatro $(\mathrm{F} 4)$, veces.

En el rendimiento en peso se incrementa en un $45 \%$ más cuándo se aplicó el Bioagroec simple (B2), respectó al testigo ( $\sin$ aplicación). En el rendimiento en peso para la categoría murmu se incrementa en un $86 \%$ más cuándo se aplicó el Bioagroec simple (B2), respectó al testigo (sin aplicación).

El análisis económico muestra que el biofertilizante (B2: con dos aplicaciones), reportó los mayores ingresos netos (1553.638 \$/ha.), y el menor costo 
variable (22.65 $\$ /$ ha). Mostrando una tasa de retorno marginal de $4181 \%$, la misma se expresa que por cada $22.65 \$$ invertidos en la producción de cultivo de papa, se recupera $41.81 \$$ adicionales. Éste tratamiento sería el más factible para su adopción por agricultores, bajo las condiciones de estudio.

\section{Conflictos de Intereses}

Esta investigación fue desarrollada por la Fundación PROINPA y no presenta conflictos de interés.

\section{Referencias citadas}

Adekiya, A. y Agbede, T. 2009. Growth and yield of tomato (Lycopersicon esculentum Mill) as influenced by poultry manure and NPK fertilizer. Emirates Journal of Food and Agriculture 21(1):10-20.

Aguilar, L. E.. 2005. Uso de caldos minerales y extractos naturales para el control del tizón tardío (Phytophthora infestans); de la papa en Colomi-Bolivia. Tesis Ing. Agro. Universidad Mayor de San Simón. Facultad de Ciencias Agrícolas y Pecuarias. CochabambaBolivia. Pág. 1-55.

Blanco, H. 2003. Efecto de la aplicación de diferentes abonos orgánicos y minerales en la producción de papa (Solanum tuberosum) en Tumuyo B Municipio de Pocona. Tesis Ing. Agr. Facultad de Ciencias Agrícolas y Pecuarias, UMSS. Cochabamba, Bolivia. p.74

Blajos, J. y Quiroga, J. 1995. Conceptos generales de costos de análisis económico. PROINPA.

Capulín, J.; Núñez, R.; Aguilar, R.; Estrada, M., Sánchez, P., Mateo, J. 2007. Uso de estiércol líquido de bovino acidulado en la producción de pimiento morrón. Revista Chapingo Serie Hortícola 13 (1):5-11.
Céspedes Y.; Álvarez, R.; Céspedes, R.; Martínez, Z. 2016. Efecto del corte y niveles de fertilización de biol en el rendimiento de materia seca y producción de semilla del pasto blando (Nasella $\mathrm{sp}$ ) con riego complementario en la Estación Experimental Choquenaira .Revista de Investigación e Innovación Agropecuaria y de Recursos Naturales, La Paz, vol. 3, n³, pág. 48-54.

ICA - Istituto ColombianoAgropecuario. 2004. Control de los Bioinsumos y Extractos Vegetales de uso agrícola en Colombia. Bogotá. En www.ica.gov.co. (Consulta: 24 de marzo 2011).

Garnica, F. 1996. Bioabonos, abonos orgánicos y un fitoestimulante en la producción de semilla de papa, en camas protegidas. Tesis Ing. Agr. Cochabamba. Facultad de Ciencias Agrícolas y Pecuarias, UMSS. Cochabamba, Bolivia. p. $51,53-54$

Macías, R.; Grijalva, R.; Robles, F. 2012. Respuesta de la aplicación de estiércol y fertilizantes sobre el rendimiento y calidad del chile jalapeño. Biotecnia 13(3):32-38.

Medina, A. 1990. "El Biol: Fuente de Fitoestimulantes en el desarrollo Agrícola". Cochabamba - Bolivia. p. 44$56,57-74$.

Medranda Vera, E.; Cedeño García, J. E.; Chávez, C.; Soplín Villacorta, H.; Lucas Vidal, L.R. 2016. Efecto del biol bovino y avícola en la producción de pimiento dulce (Capsicum annum L.). ESPAMCIENCIA 7(1): 15-2.

Nina, H.F. 2004. Rendimiento de papa con el empleo de abonos orgánicos y fertilizantes mineral en localidad de Huankuri cantón Challa Grande (Provincia Tapacari). Tesis Ing. Agr. Universidad Mayor de San Simón. Facultad de Ciencias Agrícolas y pecuarias. Cochabamba-Bolivia. Pág. 135 . 
Ortuño, N.; Navia, O.; Meneces, E.; Barja, D.; Villca, S.; Plata, G.; Claros, M.; Gutierrez, C.; Arandia, W.; Crespo, L. 2011. Catálogo de bioinsumos. Cochabamba, Bolivia. 42p.

Ramírez, L. y SOTO, G. 2000. Una experiencia de papa orgánica en la zona de Cartago, Costa Rica. En: www.infoagro.com/hortalizas. (Consulta: 18 de abril 2011).

Restrepo , J. 2002. Agricultura orgánica biofertilizantes Preparados y fermentados a base de mierda de vaca. $1^{\circ} \mathrm{Ed}$. Santiago de Cali - Colombia p.17

REDEPAPA-CORPOICA. 2002.

Fisiologia y manejo de tubérculos semilla de papa. Revista de la Asociación Brasilera de la Papa, ABBA. Traducción del portugués realizada por José D. Moreno M. [jdmoreno@corpoica.org.co], investigador del Programa Agrícola, Regional Uno, CORPOICA.

Theodoracopoulos, M.; Arias, S.; Avila, H. 2008. Manual de producción de papa. En: $\quad$ www.hondurasag.org y www.fintrac.com. (Consulta; 18 de abril 2011).

Villarroel, D. 1999. Agricultura Natural Orgánica y Ecológico. Cochabamba, Bolivia p. 23-25-28

Villarroel , J. 1886. Manual práctico para la interpretación de análisis de suelos en laboratorio. CochabambaBolivia. Serie técnica No10. p. 9-10, 14$16,21-32$. 
Tabla 1. Preparación de Biofertilizantes BioAgroec compuesto, Simple y Fertisol.

\begin{tabular}{|c|c|c|c|c|}
\hline $\begin{array}{l}\text { Insumos } \\
(100 \text { l.) }\end{array}$ & $\begin{array}{l}\text { Bioagroecc } \\
\text { ompuesto }\end{array}$ & $\begin{array}{l}\text { Bioagroec } \\
\text { simple }\end{array}$ & Fertisol & Preparación \\
\hline $\begin{array}{l}\text { Humos de lombriz } \\
\text { Estiércol fresco } \\
\text { Agua } \\
\text { Levadura } \\
\text { Garrotilla (Medicago } \\
\text { hispida) } \\
\text { Alfa alfa (Medicago } \\
\text { sativa) } \\
\text { Leche } \\
\text { Chancaca (Azúcar) } \\
\text { Trozos de metal a base } \\
\text { de hierro } \\
\text { Micronutrientes } \\
\text { Sulfato de cobre } \\
\text { Sulfato de Zinc } \\
\text { Cloruro de calcio } \\
\text { Sulfato de Magnesio } \\
\text { Macronutrientes } \\
\text { Roca fosfórica } \\
\text { Microorganismos } \\
\text { eficientes aceleradores } \\
\text { del compost. }\end{array}$ & $\begin{array}{l}- \\
23 \mathrm{~kg} . \\
77 \mathrm{l} . \\
500 \mathrm{~kg} . \\
2 \mathrm{~kg} . \\
- \\
31 . \\
2.3 \mathrm{~kg} . \\
\text { Global }\end{array}$ & $\begin{array}{l}- \\
23 \mathrm{~kg} . \\
77 \mathrm{l} . \\
500 \mathrm{gr} . \\
- \\
2 \mathrm{~kg} . \\
31 . \\
2.3 \mathrm{~kg} . \\
\text { Globa- }\end{array}$ & $\begin{array}{l}10 \mathrm{~kg} . \\
25 \mathrm{~kg} . \\
60 \mathrm{l} . \\
500 \mathrm{gr} . \\
- \\
- \\
81 . \\
3 \mathrm{~kg} . \\
--\end{array}$ & $\begin{array}{l}\text { Para el Fertisol se usó un } \\
\text { recipiente de plástico con } \\
\text { tapa de } 100 \text { lt., () } \\
\text { Donde se colocó el } \\
\text { estiércol fresco (Humos de } \\
\text { lombriz), se mezcló con el } \\
\text { agua, leche, chancaca y } \\
\text { levadura de pan disuelta en } \\
\text { agua tibia. } \\
\text { Para el Agrobioeco se } \\
\text { disolvió en un balde los } \\
\text { micro nutrientes, garrotilla } \\
\text { (alfa alfa silvestre) (solo } \\
\text { Estos componentes fueron } \\
\text { añadidos a un biol } \\
\text { corriente y fermentados } \\
\text { anaeróbicamente. } \\
\text { Revolver bien y dejar } \\
\text { fermentar entre } 1 \text { a } 3 \\
\text { meses dependiendo del } \\
\text { clima seguidamente } \\
\text { Segu se } \\
\text { cubrirá el recipiente con la } \\
\text { tapa instalada con una } \\
\text { manguera de plástico junto } \\
\text { a la botella. }\end{array}$ \\
\hline
\end{tabular}

Tabla 2. Costos de inversión, beneficios netos, dominancia y tasa de retorno marginal en la producción del cultivo de la papa.

\begin{tabular}{|c|c|c|c|c|c|c|}
\hline Tratamiento & $\begin{array}{l}\text { Costos variables } \\
\text { (CV) \$ }\end{array}$ & $\begin{array}{l}\text { Ingresó neto } \\
\text { (IN) \$ }\end{array}$ & Dominancia & $\square(\mathbf{C V}) \mathbf{S}$ & $\square$ (IN) $\mathbf{S}$ & $\begin{array}{l}\text { TRM } \\
(\%)\end{array}$ \\
\hline $\mathrm{T} 1=\mathrm{B} 0 \mathrm{~F} 0$ & 0.00 & 606.68 & ND & & & \\
\hline $\mathrm{T} 5=\mathrm{B} 2 \mathrm{~F} 2$ & 22.65 & 1553.638 & ND & 22.65 & 946.958 & 4181 \\
\hline $\mathrm{T} 2=\mathrm{B} 1 \mathrm{~F} 2$ & 25.10 & 580.06 & SD & & & \\
\hline $\mathrm{T} 6=\mathrm{B} 2 \mathrm{~F} 3$ & 34.95 & 1295.25 & SD & & & \\
\hline $\mathrm{T} 3=\mathrm{B} 1 \mathrm{~F} 3$ & 38.80 & 718.36 & SD & & & \\
\hline $\mathrm{T} 7=\mathrm{B} 2 \mathrm{~F} 4$ & 47.25 & 1304.23 & SD & & & \\
\hline $\mathrm{T} 4=\mathrm{B} 1 \mathrm{~F} 4$ & 52.50 & 714.692 & SD & & & \\
\hline $\mathrm{T} 8=\mathrm{B} 3 \mathrm{~F} 2$ & 74.63 & 594.375 & SD & & & \\
\hline $\mathrm{T} 9=\mathrm{B} 3 \mathrm{~F} 3$ & 116.63 & 1035.735 & SD & & & \\
\hline $\mathrm{T} 10=\mathrm{B} 3 \mathrm{~F} 4$ & 158.63 & 1262.775 & SD & & & \\
\hline
\end{tabular}




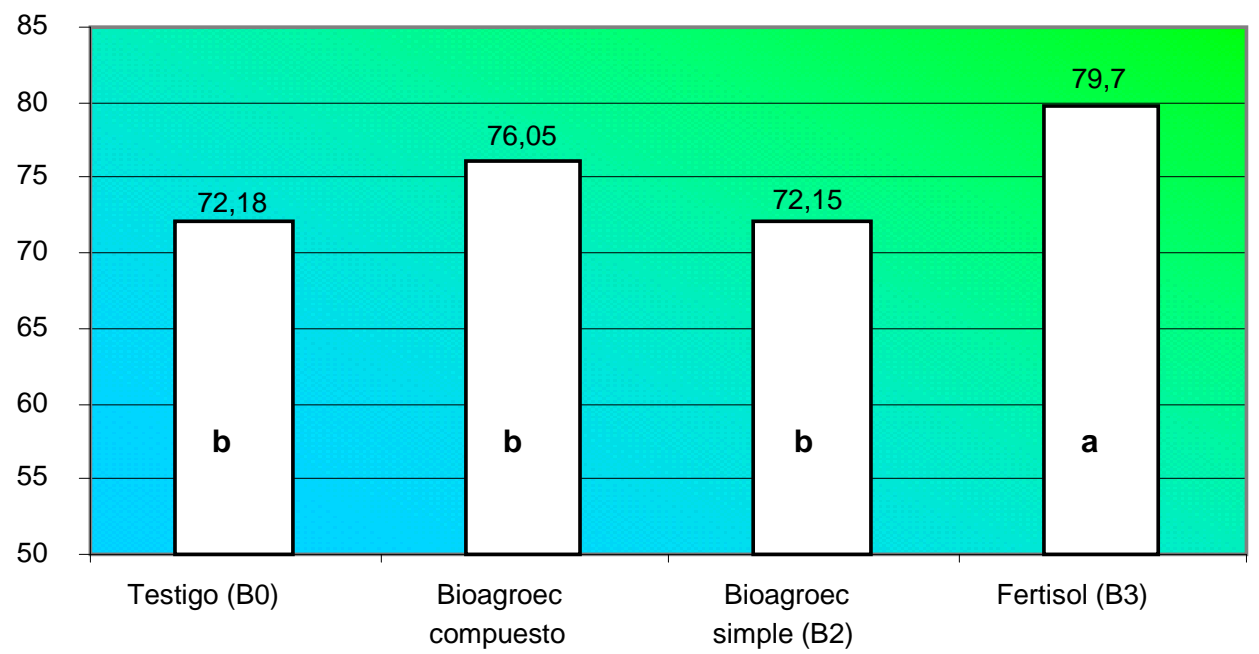

(B1)

Biofertilizantes

Figura 1. Efecto de los biofertilizantes sobre altura planta en el cultivo de papa Huaycha en el Municipio de Pocona-Cochabamba.

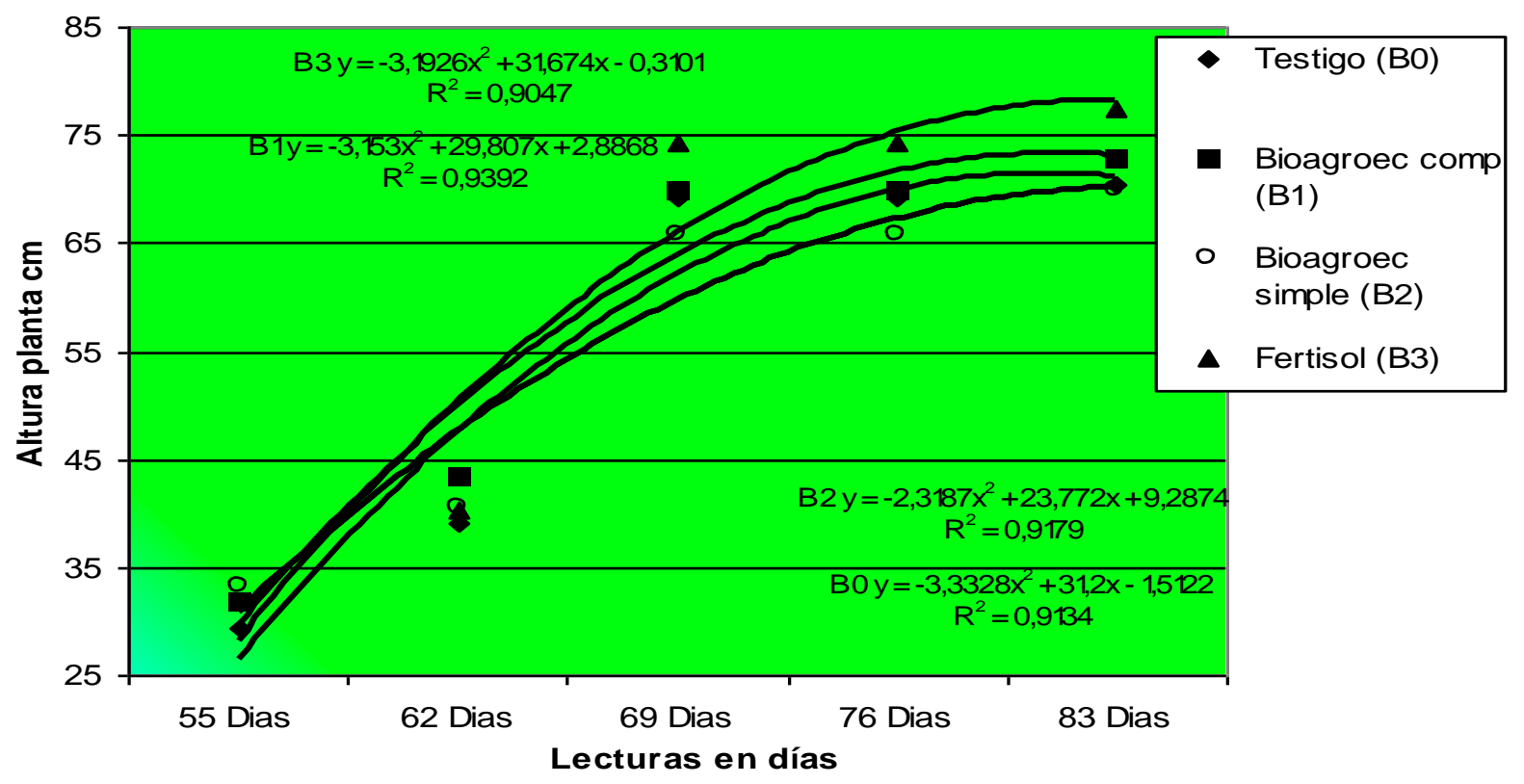

Figura 2. Efecto de las frecuencias de aplicación de los Biofertilizantes cuando se aplica cuatro veces (a los 55, 67, 79 y 91 días), en todo el ciclo del cultivo de papa. 


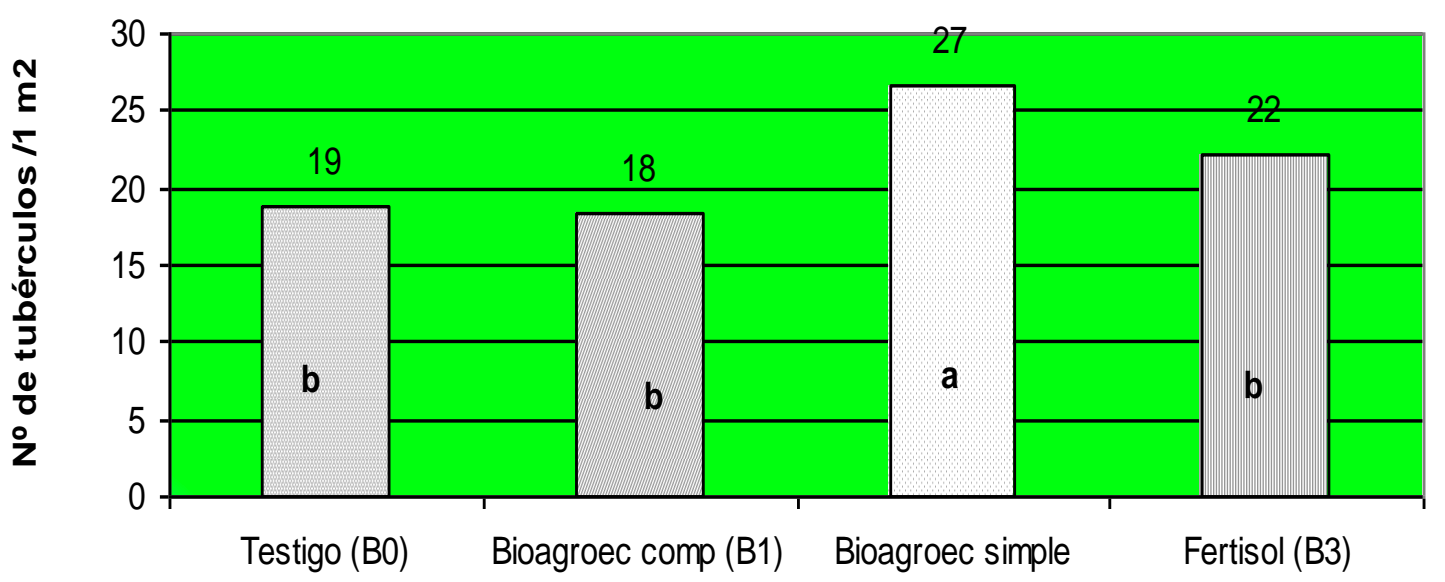

(B2)

\section{Biofertilizantes}

Figura 3. Comparación de medias en la variable número de tubérculos por tratamiento en el cultivo de papa Huaycha.

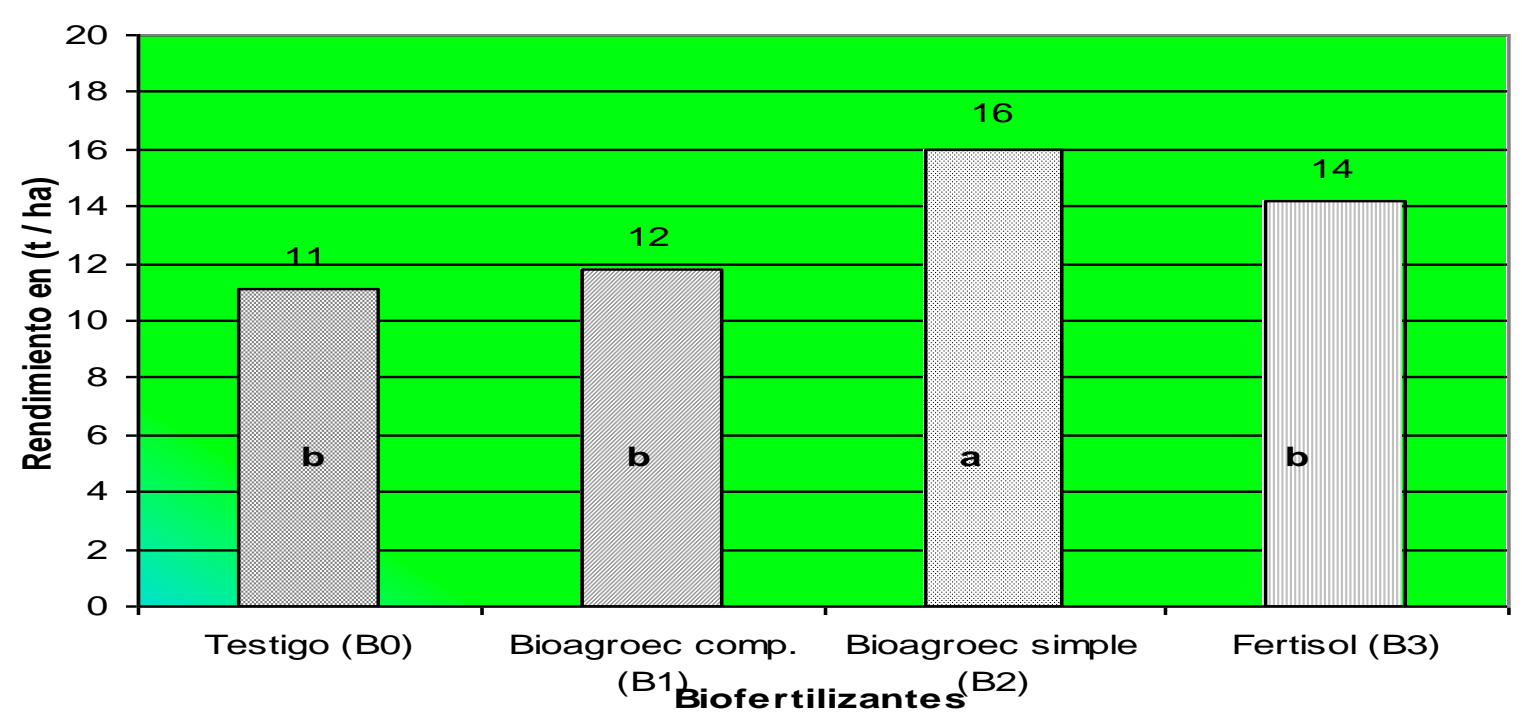

Figura 4. Comparación de medias entre Biofertilizantes y el testigo en el rendimiento total en peso del cultivo papa Huaycha. 